\title{
AEROSOL/CLOUD MEASUREMENTS USING COHERENT WIND DOPPLER LIDARS
}

\author{
Philippe Royer $^{1 *}$, Matthieu Boquet ${ }^{1}$, Jean-Pierre Cariou ${ }^{1}$, Laurent Sauvage ${ }^{1}$, Rémy Parmentier ${ }^{1}$ \\ ${ }^{1}$ LEOSPHERE, 14-16 rue Jean Rostand, 91400, Orsay, FRANCE, *Email: proyer@leosphere.com
}

\begin{abstract}
The accurate localization and characterization of aerosol and cloud layers is crucial for climate studies (aerosol indirect effect), meteorology (Planetary Boundary Layer PBL height), site monitoring (industrial emissions, mining,...) and natural hazards (thunderstorms, volcanic eruptions).

LEOSPHERE has recently developed aerosol/cloud detection and characterization on WINDCUBE long range Coherent Wind Doppler Lidars (CWDL). These new features combine wind and backscatter intensity informations (Carrier-to-Noise Ratio CNR) in order to detect (aerosol/cloud base and top, PBL height) and to characterize atmospheric structures (attenuated backscatter, depolarization ratio). For each aerosol/cloud functionality the method is described, limitations are discussed and examples are given to illustrate the performances.
\end{abstract}

\section{INTRODUCTION}

Coherent Lidars are nowadays well-recognized for their accurate measurements of wind speed profiles based on the Doppler shift effect on atmospheric particles and clouds. The signal reflectivity (CNR for Carrier-to-Noise Ratio) profiles can also be retrieved from the signal strength of the detected Doppler peak.

Based on the CNR and wind information, specific aerosol and cloud functionalities were developed on WINDCUBE long range scanning Doppler lidar at 1.54 microns. They consist in 6 aerosol/cloud features:

1) Aerosol/cloud detection

2) Aerosol/cloud discrimination

3) Attenuated backscatter coefficient

4) Planetary Boundary Layers height detection
5) Depolarization ratio

6) Speciation of atmospheric structures

The general flowchart of the aerosol/cloud measurements is given on figure 1. Each function is described and discussed in the next section

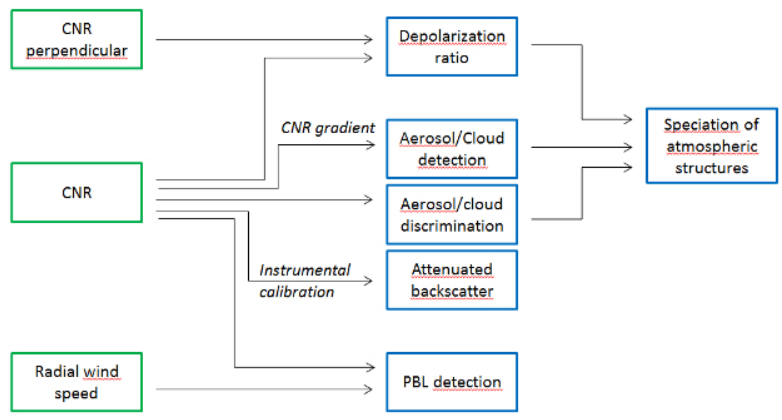

Figure 1: General flowchart of the aerosol/cloud measurements on WINDCUBE lidars

\section{RESULTS}

\subsection{AEROSOL/CLOUD DETECTION}

The layer detection is based on automated detection of gradient on CNR signal. Figure 2 shows the three steps to detect base and top of each atmospheric layer:

- Step1 : CNR signal is filtered and the gradient is computed using a Sobel mask

- Step 2 : The gradient are automatically segmented using an OTSU method [1]

- Step 3 : Base and top of atmospheric layers are identified

CWDL aerosol/cloud detection has been compared the aerosol lidar (R-MAN510) and showed quite similar results (see figure 3 ). 


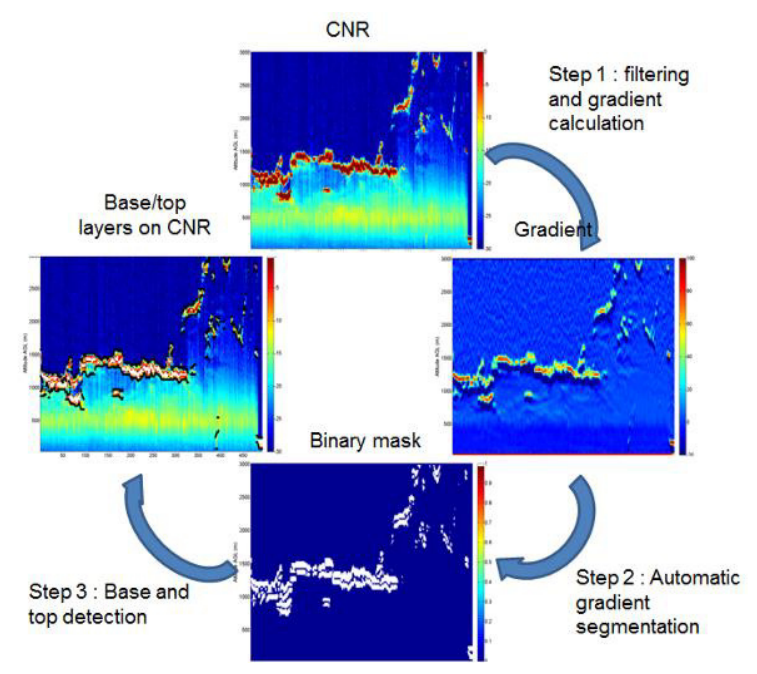

Figure 2: Automatic detection of base and top of atmospheric layers based on CNR signal
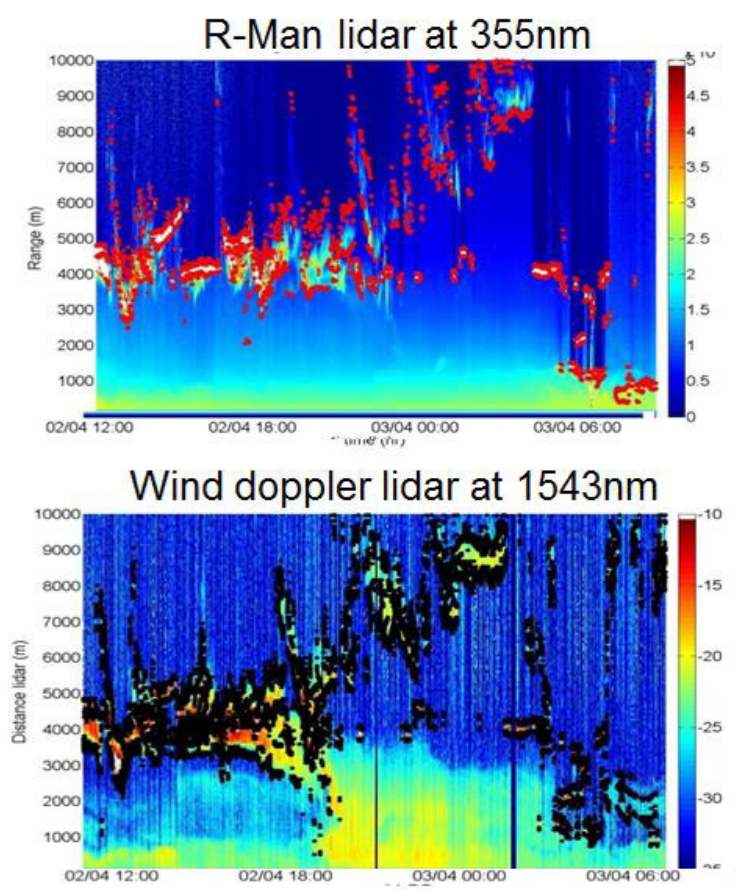

Figure 3: Comparison of base and top of atmospheric layers detected with an aerosol lidar at 355nm (red dots on the top panel) and a CWDL at $1543 \mathrm{~nm}$ (black dots on the bottom panel)

\subsection{AEROSOL/CLOUD DISCRIMINATION}

Each detected structure is discriminate in aerosol of cloud based on CNR peak intensity. The probability of aerosol and cloud is estimated with the following steps:
- compute average CNR gradient in layer between base and top

- estimate probability of presence aerosol and cloud using value of gradient and statistical results from WINDCUBE200S campaigns

- discriminate layer into aerosol or cloud using probability of presence and neighborhood information

Examples of aerosol/cloud discrimination are given on figure 4 in PPI (Plan Position Indicator) and fixed LOS (Line Of Sight) measurements.

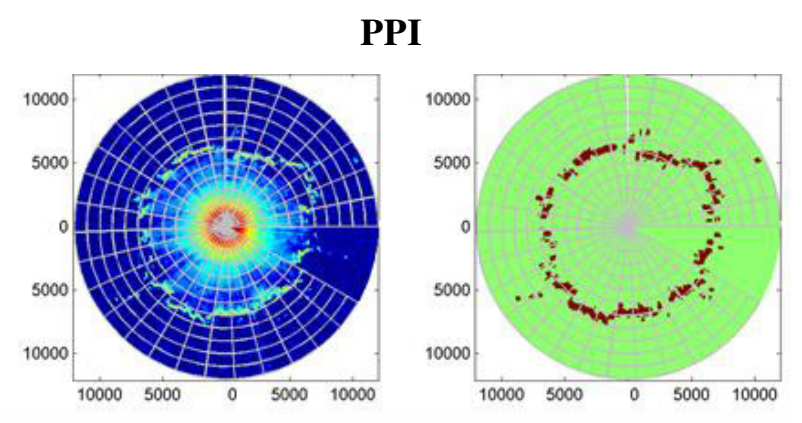

Fixed LOS
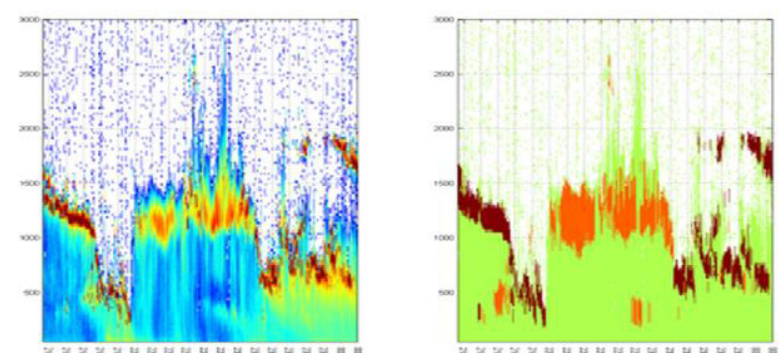

Figure 4: Examples of CNR signals (left panels) and aerosol (orange) and cloud (brown) discrimination (right panels) in PPI (top panel) and fixed LOS (bottom panel)

\subsection{ATTENUATED BACKSCATTER}

The attenuated backscatter coefficient is a function of the aerosol type and concentration. This parameter can be used to highlight aerosol and cloud layer in the atmosphere. An automatic calibration is performed every hour to compute and remove the instrumental function of the instrument. 
Figure 5 shows the comparison of attenuated backscatter coefficient retrieved with a WINDCUBE200S lidar at $1543 \mathrm{~nm}$ and the absolute backscatter coefficient at $355 \mathrm{~nm}$ from the R-MAN510 aerosol lidar.
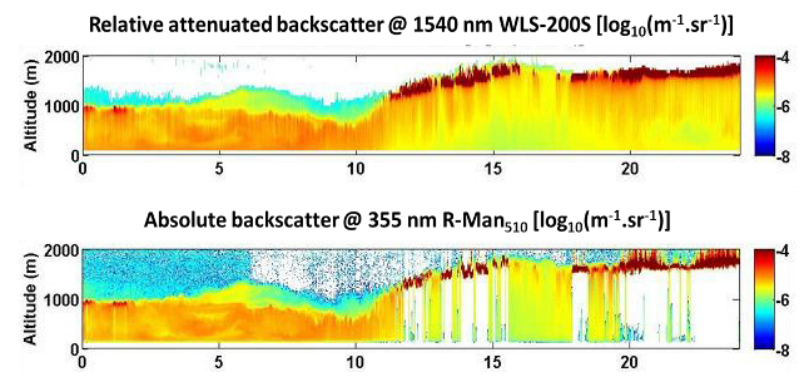

Figure 5: Comparison of attenuated backscatter coefficient retrieved from a CWDL at $1543 \mathrm{~nm}$ (top panel) and an aerosol lidar (R-MAN510) at $355 \mathrm{~nm}$ (bottom panel)

\subsection{PLANETARY BOUNDARY LAYER HEIGHT}

Residual and mixing layers detections are described on figure 6 .
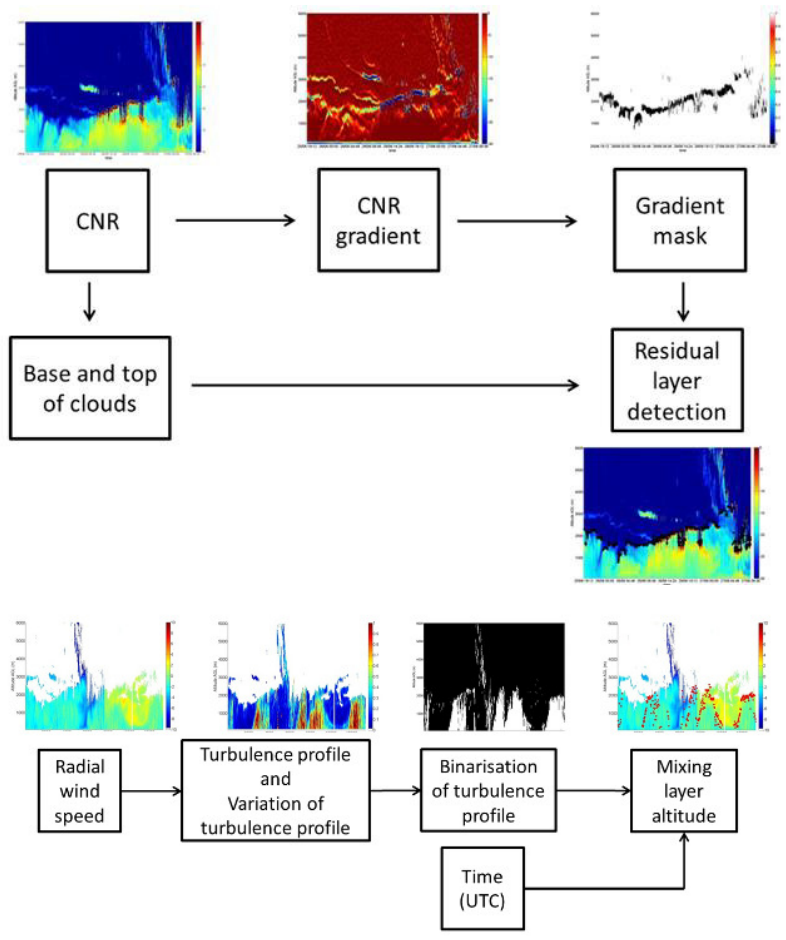

Figure 6: Residual (top panel) and mixing (bottom panel) layer detections on WINDCUBE long range lidars
The residual layer is the transition between aerosol in the boundary layer and the free troposphere. The residual layer is bounded above by a capping inversion. The residual layer is retrieved using CNR gradient (top panel on figure 7).

Mixing layer is the layer where positive buoyancy flux at the surface creates a thermal instability and thus generates additional or even major turbulence. Mixing layer is thus retrieved using turbulence information determined by vertical wind dispersion (bottom panel on figure 7, [2]).
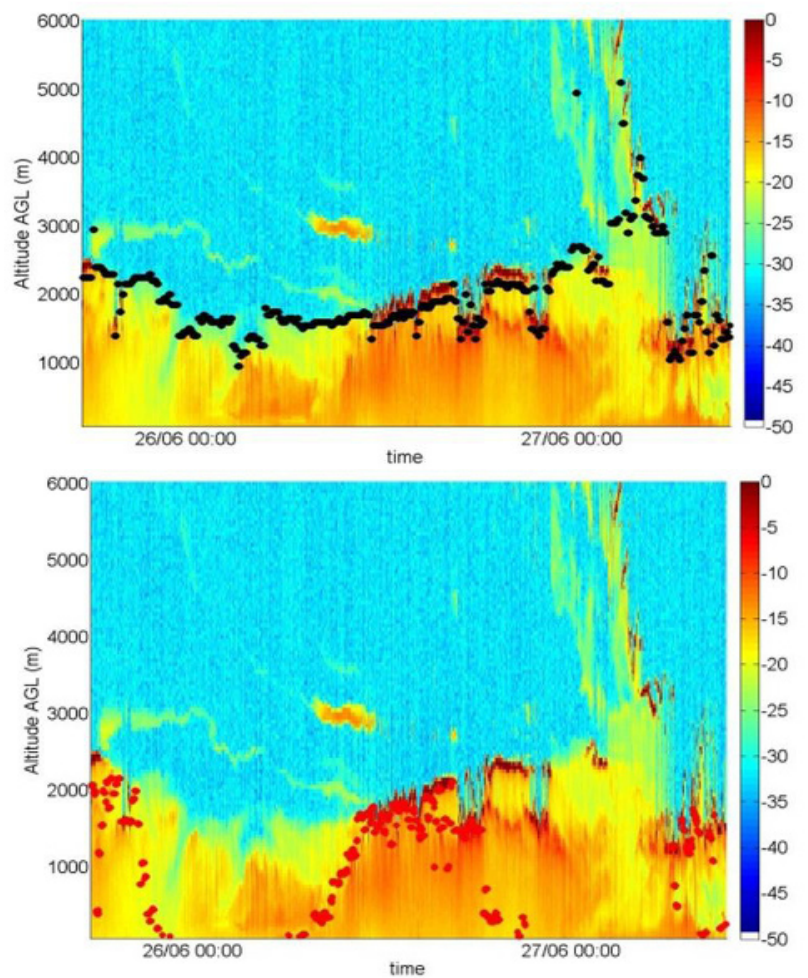

Figure 7: Example of residual layer (black dots on top panel) and mixing layer detection (red dots on bottom panel)

\subsection{DEPOLARIZATION RATIO}

Depolarization ratio gives information on particle shape. High depolarization ratio are expected for non-spherical scatterers (ice clouds, dust, ashes) whereas low depolarization are expected from spherical particles (sea salt, pollution, biomass burning) or water-clouds. Depolarization ratio is computed at $1543 \mathrm{~nm}$ using information from copolar and cross-polar CNR signals. 
Dust event has been measured with high depolarization ratio in the boundary layer (near to $30 \%$, figure 8 ). This case is referenced in the operational website of the BSC-DREAM8b model [3] with a maximum concentration of $50 \mu \mathrm{g} / \mathrm{m}^{3}$ over Paris (figure 8). Water clouds and ice clouds have been also been observed at the same time at the top and above the PBL.

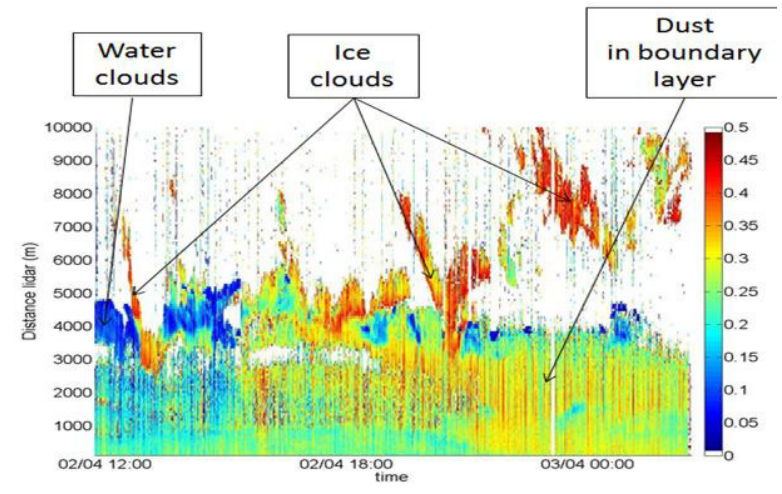

Figure 8: Depolarization measurement during a dust event observed over Paris the $2^{\text {nd }}$ of April 2014

\subsection{SPECIATION OF ATMOSPHERIC STRUCTURES}

The mean value of depolarization ratio gives information on shape and type of each aerosol and cloud layer detected (figure 5). Combining information from aerosol/cloud detection and discrimination and depolarization measurement the atmospheric structures are classified into 6 types: aspherical aerosols (ash, dusts), spherical aerosols (sea salt, biomass burning, continental pollution), water clouds (water droplets), ice clouds (e.g. cirrus clouds), unclassified aerosol and unclassified clouds.

An example of atmospheric structure speciation from WINDCUBE200S measurements is given on figure 9.
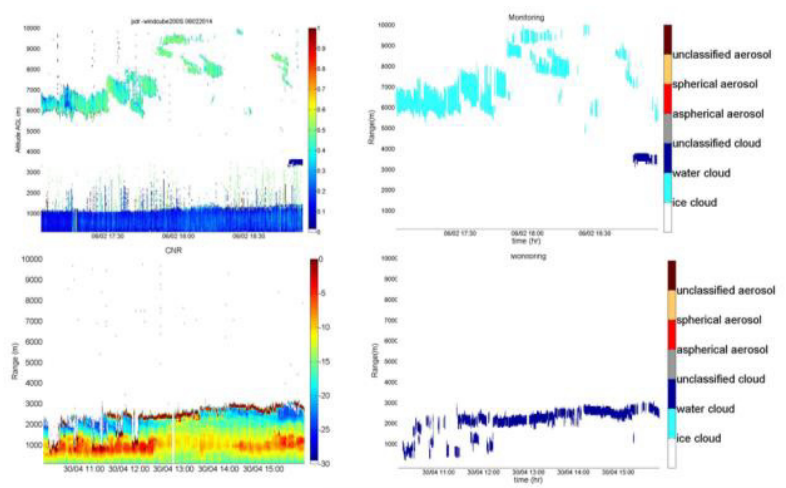

Figure 9: Example of atmospheric structure speciation for ice (top) and water clouds (bottom)

\section{CONCLUSIONS}

Based on wind and CNR information, aerosol and cloud capabilities have been developed on CWDL for the detection and the characterization of atmospheric structures. The results have been compared with aerosol lidar retrievals and showed similar performances.

The main benefit of CWDL technology is the ability to retrieve both wind (direction and intensity) and aerosol/cloud information. This ability is particularly important for the monitoring of the dispersion on industrial sites, the understanding of atmospheric hazard (thunderstorms formation) and the air traffic survey (identification and localization of volcanic ashes).

\section{REFERENCES}

[1] Otsu, N., 1979: A threshold selection method from gray-level histograms, IEEE Trans. Sys., Man., Cyber., vol. 9, 1979, p. 62-66

[2] Bianco, L., Wilczak, J. M., White, A. B., 2008: Convective Boundary Layer Depth Estimation from Wind Profilers: Statistical Comparison between an Automated Algorithm and Expert Estimations, J. Tech. A, DOI: 10.1175/2008JTECHA981.1, 1397-1413.

[3] Basart et al., 2012: Development and evaluation of the BSC-DREAM8b dust regional model over Northern Africa, the Mediterranean and the Middle East, Tellus B, 64, 18539, doi:10.3402/tellusb.v64i0.18539. 